\title{
Size-dependent interface phonon transmission and thermal conductivity of nanolaminates
}

\author{
L. H. Liang, ${ }^{1,2, a)}$ Y. G. Wei, ${ }^{1}$ and Baowen $\mathrm{Li}^{2}$ \\ ${ }^{1}$ LNM, Institute of Mechanics, Chinese Academy of Sciences, Beijing 100080, People's Republic of China \\ ${ }^{2}$ Department of Physics and Centre for Computational Science and Engineering, National University \\ of Singapore, Singapore 117542, Singapore
}

(Received 26 October 2007; accepted 23 February 2008; published online 29 April 2008)

\begin{abstract}
An analytical model for size-dependent interface phonon transmission and thermal conductivity of nanolaminates is derived based on the improved acoustic mismatch theory and the Lindemann melting theory by considering the size effect of phonon velocity and the interface lattice mismatch effect. The model suggests that the interface phonon transmission is dominant for the cross-plane thermal conductivity of nanolaminates and superlattices, and the intrinsic variety of size effect of thermal conductivity for different systems is proposed based on the competition mechanism of size effect of phonon transport between two materials constituting the interfaces. The model's prediction for thermal conductivity of nanolaminates agrees with the experimental results. (C) 2008 American Institute of Physics. [DOI: 10.1063/1.2910828]
\end{abstract}

\section{INTRODUCTION}

With a continuous miniaturization of microelectronic devices, heat dissipation and other thermal management problems become more and more important. ${ }^{1-3}$ Some devices such as computer processors and integrated circuits need high thermal conductivity, which is favorable to get the heat away. On the other hand, for thermal barriers and thermoelectric devices, low thermal conductivity is desired. The composite nanolaminates with high interface intensity have potential application for improving the performance of these devices. The interface thermal resistance between different materials exists since the interface breaks the regular lattice structure; the interface phonon transport plays more and more important role in thermal conductivity of nanolaminates and superlattices as the interface distance or periodic thickness reduces.

The interface thermal resistance has been studied since half century before. The earliest theoretical explanation for interface resistance is the acoustic mismatch theory, ${ }^{4}$ in which the interface phonon transmission efficiency is captured by the acoustic impedance of materials at the two sides of the interface. Another classic investigation is the diffuse mismatch theory, ${ }^{5}$ assuming that the probability of phonons being scattered to any side of the interface is simply proportional to the phonon density of states. However, both theories are based on the consideration of the bulk. For nanoscale materials and interfaces, the related physical properties such as phonon velocity and thermal conductivity are different from the corresponding bulks. Moreover, both theories are independent of the structure of the interface itself. What is the actual case for interface phonon transport of nanoscale systems?

In this work, a theoretical model about size-dependent interface phonon transmission and thermal conductivity for nanolaminates is proposed based on the modified acoustic

${ }^{a)}$ Electronic mail: lianglh@lnm.imech.ac.cn. mismatch theory by introducing the intrinsic size effect of phonon velocity and the interface structure mismatch effect. The theory predicts the variety of size dependence of interface phonon transmission for different systems and explains the variety of size dependence of thermal conductivity of different superlattices. This variety as the intrinsic characteristics of interfaces has not been recognized in previous study. Based on the consideration of the interface transport dominance, the thermal conductivity of $\mathrm{W} / \mathrm{Al}_{2} \mathrm{O}_{3}$ nanolaminates is calculated, and the results agree with the experimental evidence.

\section{MODEL AND DISCUSSION}

\section{A. Size-dependent interface phonon transmission}

According to the acoustic mismatch theory, the transmission coefficient $t_{A B}$ of phonon propagation through the interface between $A$ and $B$ materials is expressed as ${ }^{2,4}$

$$
t_{A B}=4 Z_{A} Z_{B} /\left(Z_{A}+Z_{B}\right)^{2},
$$

where $Z=\rho v$ is the acoustic impedance, $\rho$ the mass density, and $v$ the phonon velocity. The subscripts $A$ and $B$ represent the respective materials at two sides of the interface. The phonon velocity is size dependent for nanomaterials, ${ }^{2}$ the size dependence of $v$ for $A$ and $B$ materials should contribute to their acoustic impedance and hence induce the size effect of the interface phonon transmission.

According to the expression for determining the characteristic Debye temperature of solids $\Theta, \Theta=\left[2 h_{p} /\left(\pi k_{B}\right)\right]$ $\times\left[3 N_{A} /(4 \pi V)\right]^{1 / 3} v$ with the Planck constant $h_{P}$, the Boltzmann constant $k_{B}$, the Avogadro constant $N_{A}$, and the molar volume $V,{ }^{6} v$ is proportional to $\Theta$. Assuming that the above equation is valid for the corresponding nanosolids, let $D$ be the size of nanosolids, such as the diameter of nanoparticles or the thickness of thin films, $\infty$ corresponds to the bulk limit, the size dependence of $v$ is equal to that of $\Theta, v(D) / v(\infty)$ $=\Theta(D) / \Theta(\infty)$. According to the Lindemann melting criterion and the Einstein specific heat theory, the square of the char- 


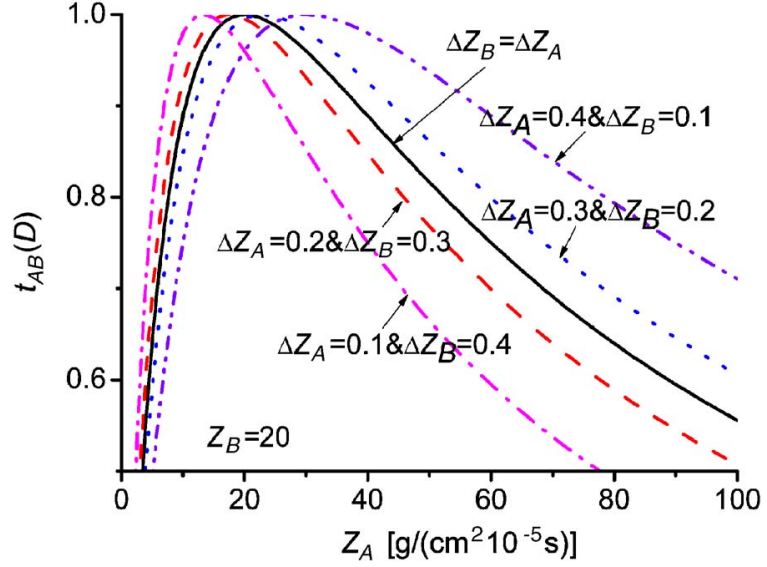

FIG. 1. (Color online) $t_{A B}(D)$ vs $Z_{A}$ based on Eqs. (3) and (1). $Z_{B}(\infty)$ $=20 \mathrm{~g} / \mathrm{cm}^{2} 10^{-5} \mathrm{~s}$ is given as the reference of calculation.

acteristic temperature is proportional to the melting temperature $T_{m}$ of solids, $\Theta=$ const. $\left[T_{m} /\left(M V^{2 / 3}\right)\right]^{1 / 2}$, with the molecular weight $M .^{7}$ Assuming the same relation for the nanosolids, $\Theta(D) / \Theta(\infty)=\left[T_{m}(D) / T_{m}(\infty)\right]^{1 / 2}$. Therefore, the size dependence of $v$ can be obtained by that of $T_{m}$, $v(D) / v(\infty)=\left[T_{m}(D) / T_{m}(\infty)\right]^{1 / 2}$. Based on the Lindemann melting criterion and the size-dependent atomic thermal vibration for nanocrystals, the size-dependent melting temperature function has been given as ${ }^{8}$

$$
T_{m}(D) / T_{m}(\infty)=\exp \left[(1-\alpha) /\left(D / D_{0}-1\right)\right],
$$

where $\alpha=2 S_{v} /(3 R)+1$ is a material constant with the bulk vibration entropy of melting $S_{v}$ and the ideal gas constant $R{ }^{8}$ $D_{0}=2(3-d) h$ is a critical size, $h$ the atomic or molecular diameter, and the dimensions $d=0,1$, and 2 for nanoparticles, nanowires, and thin films, respectively. ${ }^{8}$ Finally,

$$
v(D) / v(\infty)=\left\{\exp \left[(1-\alpha) /\left(D / D_{0}-1\right)\right]\right\}^{1 / 2} .
$$

Substituting Eq. (3) for $A$ and $B$ into Eq. (1), respectively, the interface phonon transmission with the intrinsic size effect $t_{A B}(D)$ can be obtained corresponding to the interface or period thickness $D$. Nevertheless, Eq. (1) merely joins two materials but omits the properties of the interface itself, which also affect the transmission efficiency. Therefore, we further consider the effect of intrinsic interface structure by introducing the lattice mismatch factor $f=\left(a_{A}\right.$ $\left.-a_{B}\right) / a_{B}$ since the lattice mismatch contributes to the interface resistance, ${ }^{9}$ where $a$ is the lattice constant and $a_{A}>a_{B}$. Adding the interface structure influence to the phonon transmission, as the first order approximation, the effective phonon transmission coefficient $t_{e}$ can be expressed as

$$
t_{e}=(1-f) t_{A B}(D) \text {. }
$$

Figure 1 shows the size-dependent interface phonon transmission $t_{A B}(D)$ and the variety of size effect in different cases. Let $\Delta Z_{i}(i=A, B)=\left[Z_{i}(\infty)-Z_{i}(D)\right] / Z_{i}(\infty)$ represents the change of acoustic impedance of materials due to the drop of phonon velocity, it can be seen from the figure that when $Z_{A}(\infty)>Z_{B}(\infty)$ (see the side of $Z_{A}>20$ ) and the decrease of acoustic impedance of $A$ is larger than that of $B$ (see the above of the solid curve, $\Delta Z_{A}>\Delta Z_{B}$ ), the interface transmission increases [i.e., $t_{A B}(D)>t_{A B}(\infty), t_{A B}(\infty)$ corresponds to the solid curve]; contrarily, the transmission decreases $\left[t_{A B}(D)<t_{A B}(\infty)\right]$ at $\Delta Z_{A}<\Delta Z_{B}$, but $t_{A B}$ increases with the enhancement of $\Delta Z_{A}$ or the decrease of $\Delta Z_{B}$ in both cases. When $Z_{A}(\infty)<Z_{B}(\infty)$, vice versa. The variety of size effects comes from the competition of the size dependences of phonon transport of two materials constituting the interface.

Figure 2(a) shows the effective interface phonon transmission $t_{e}$ for some real systems. The variety of size dependence of $t_{e}$ for different systems can also be found. For some interfaces such as $\mathrm{GaAs} / \mathrm{AlAs}, \mathrm{W} / \mathrm{Al}_{2} \mathrm{O}_{3}$, etc., $t_{e}$ monotonously decreases with reducing interface thickness $D$. For others, the case is different. For example, for $\mathrm{Si} / \mathrm{Ge}$ interface, $t_{e}$ increases with reducing $D$; but when $D$ reduces to near the critical size, $t_{e}$ abruptly decreases, showing a peak behavior, which may attribute to the resonance tunneling caused by ballistic phonon transport. Although the obvious size effect of $t_{e}$ appears at the smaller size, the properties of this size range play an important role in the performance of nanoscale devices. The increase or decrease of the phonon transmission and thermal conduction with reducing size can be designed by using the variety of size effects of different systems.

As a comparison, the experimental results of cross-plane thermal conductivity $\kappa$ for $\mathrm{Si} / \mathrm{Ge}, \mathrm{GaAs} / \mathrm{AlAs}$ superlattices, and $\mathrm{W} / \mathrm{Al}_{2} \mathrm{O}_{3}$ nanolaminates are shown in Figs. 2(b) and 2 (c), respectively. Figure 2(b) shows that $\kappa$ of $\mathrm{Si} / \mathrm{Ge}$ superlattices increases with reducing period $D$ for most measurements, especially, one set of data shows the peak behavior; ${ }^{22}$ the tendency agrees with that for $t_{e}$. Only exceptive observation may be due to the break of superlattice structure at the given size, ${ }^{25}$ but $\kappa$ increases with reducing $D$ in both ranges of larger and smaller than the size. It also shows that $\kappa$ of GaAs/AlAs superlattices [Fig. 2(b)] and $\mathrm{W} / \mathrm{Al}_{2} \mathrm{O}_{3}$ nanolaminates [Fig. 2(c)] decrease with reducing period or interface distance $D$; the tendency also agrees with that for $t_{e}$. The agreement implies that the interface phonon transmission dominates the thermal conductivity, especially for the thinner laminates. To validate further, we discuss the thermal conductivity of $\mathrm{W} / \mathrm{Al}_{2} \mathrm{O}_{3}$ nanolaminates.

\section{B. Thermal conductivity of nanolaminates}

The interface conductance $G$, related to the interface phonon transmission, is assumed to dominate $\kappa$ of $\mathrm{W} / \mathrm{Al}_{2} \mathrm{O}_{3}$ since the laminates are very thin, ${ }^{3} \quad \kappa=G l=\left(\right.$ const $\left.v C t_{e}\right) l$ $=\left(\right.$ const $\left.^{\prime} v C l^{\prime}\right) t_{e}=\kappa_{0} t_{e}$ based on the definition of the interface conductance 2,27 and the kinetic formula of thermal conductivity, ${ }^{2}$ where $l$ denotes the Kapitza length, $C$ is the specific heat, $l^{\prime}$ is the mean free path of phonons, and the imaginary thermal conductivity $\kappa_{0}$ is introduced by the above transition, ${ }^{5}$ which is related to the interface thermal conductivity without the transmission efficiency consideration corresponding to a certain thickness of the available minimum interface distance $D_{\min }$ and can be obtained by averaging the thermal conductivity of two materials constituting the interface. Note that the interface in ideal case is a plane, but the actual interface has the certain thickness, thus $D_{\min }$ is taken as the thickness approximately. In the experiment of $\mathrm{W} / \mathrm{Al}_{2} \mathrm{O}_{3}, D_{\min }$ is about $1.6 \mathrm{~nm},{ }^{3}$ thus the thicknesses of $\mathrm{W}$ 

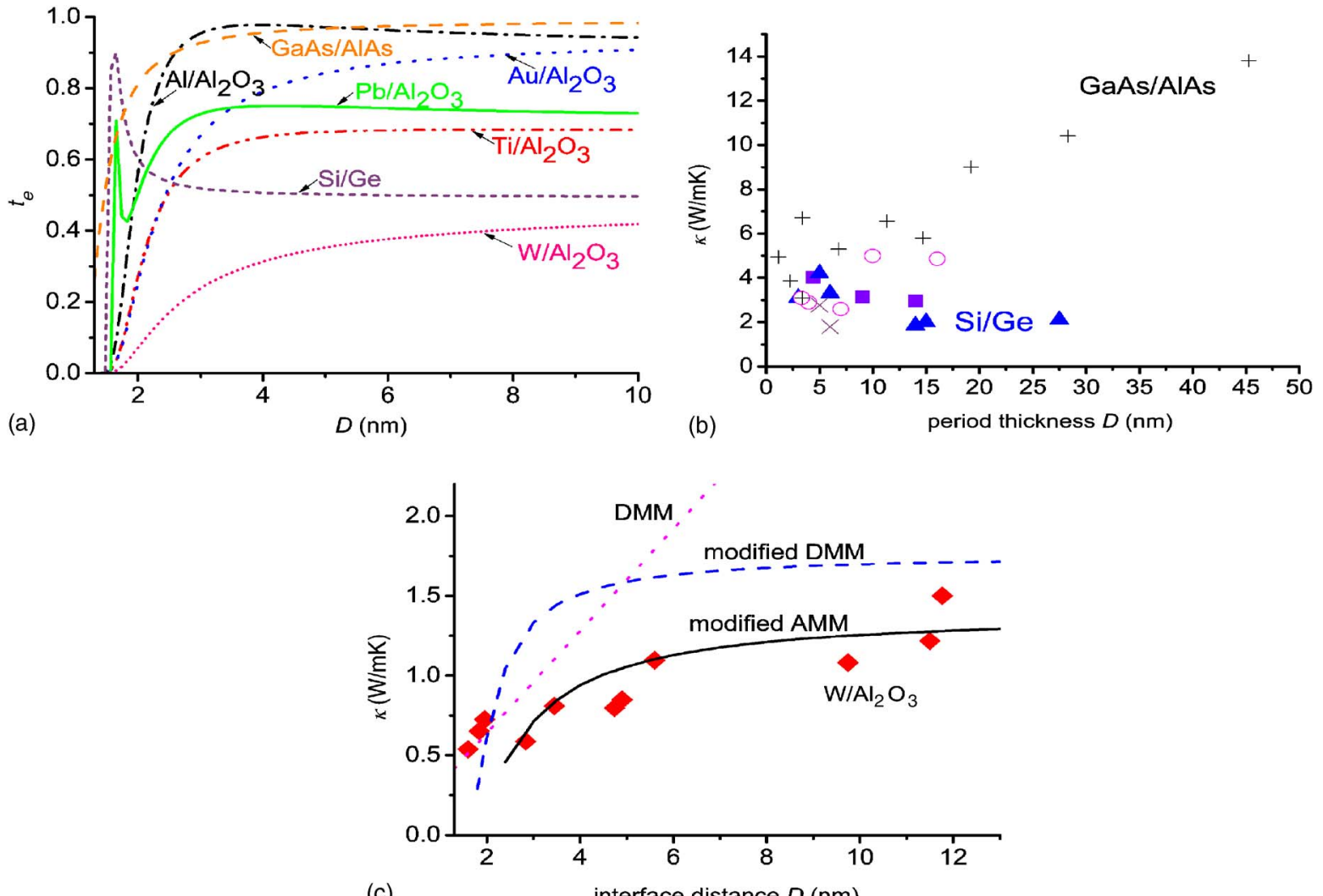

(c)

interface distance $D(\mathrm{~nm})$

FIG. 2. (Color online) (a) $t_{e}$ vs $D$ based on Eq. (4). The related parameters see Table I. (b) Measured $\kappa$ of Si/Ge and GaAs/AlAs supperlattices at room temperature. The triangles, squares, forks, and circles represent the data of $\mathrm{Si} / \mathrm{Ge}$ cited from Refs. 22-25; the crosses for GaAs/AlAs (Ref. 26) (c) $\kappa$ of $\mathrm{W} / \mathrm{Al}_{2} \mathrm{O}_{3}$ nanolaminates. The rhombuses are experimental data (Ref. 3). The solid curve is our model's prediction, in Eq. $(5), \kappa(\infty)=174$ and $35 \mathrm{~W} / \mathrm{m} \mathrm{K}$ for $\mathrm{W}$ (Ref. 11) and $\mathrm{Al}_{2} \mathrm{O}_{3}$ (Ref. 13). The dashed and dotted curves are DMM predictions, the dotted curve for the constant $G=320 \mathrm{MW} / \mathrm{m}^{2} \mathrm{~K}$ and $\kappa=G D$ (Refs. 3 and 5), the dashed one for the modified transmission and conductivity, $C=132$ and $419 \mathrm{~J} / \mathrm{kg} \mathrm{K}$ for W (Ref. 11) and $\mathrm{Al}_{2} \mathrm{O}_{3}(\operatorname{Ref}$ 30).

TABLE I. The bulk parameters in the model. In Eq. (3), $D_{0}=2 h, \alpha$ $=2 S_{v} /(3 R)+1$, and $S_{v}=S_{m}$ for metals (Ref. 8), where the melting entropy $S_{m}=H_{m} / T_{m}$ with the melting enthalpy $H_{m}$; for semiconductors and dielectrics, $S_{v}=S_{m}-R$ (Ref. 10).

\begin{tabular}{lcccccc}
\hline \hline & $\begin{array}{c}\rho \\
\left(\mathrm{g} / \mathrm{cm}^{3}\right)^{\mathrm{a}}\end{array}$ & $v(\mathrm{~m} / \mathrm{s})^{\mathrm{b}}$ & $a(\mathrm{~nm})^{\mathrm{c}}$ & $\begin{array}{c}H_{m} \\
(\mathrm{KJ} / \mathrm{mol})^{\mathrm{a}}\end{array}$ & $T_{m}(\mathrm{~K})^{\mathrm{a}}$ & $h(\mathrm{~nm})^{\mathrm{d}}$ \\
\hline $\mathrm{W}$ & 19.3 & 5174 & 0.3165 & 35.4 & 3680 & 0.3116 \\
$\mathrm{~Pb}$ & 11.4 & 1260 & 0.495 & 4.799 & 600.6 & 0.387 \\
$\mathrm{Ti}$ & 4.5 & 4140 & 0.295 & 15.45 & 1943 & 0.323 \\
$\mathrm{Al}$ & 2.7 & 5100 & 0.405 & 10.79 & 933.25 & 0.3164 \\
$\mathrm{Au}$ & 19.3 & 1740 & 0.408 & 12.55 & 1337.58 & 0.3198 \\
$\mathrm{Si}$ & 2.33 & 2200 & 0.543 & 50.55 & 1685 & 0.3368 \\
$\mathrm{Ge}$ & 5.32 & 5400 & 0.5657 & 36.94 & 1210.4 & 0.351 \\
$\mathrm{Al} \mathrm{O}_{3}$ & $4^{\mathrm{b}}$ & $6000^{\mathrm{e}}$ & $0.395^{\mathrm{e}}$ & $111.4^{\mathrm{c}}$ & $2326^{\mathrm{c}}$ & $0.324^{\mathrm{f}}$ \\
$\mathrm{GaAs}$ & $5.32^{\mathrm{c}}$ & $3859^{\mathrm{g}}$ & 0.5653 & $120^{\mathrm{h}}$ & $1511^{\mathrm{h}}$ & $0.2448^{\mathrm{i}}$ \\
$\mathrm{AlAs}$ & $3.81^{\mathrm{c}}$ & $6440^{\mathrm{j}}$ & 0.5662 & $119.78^{\mathrm{k}}$ & $2013^{\mathrm{k}}$ & $0.245^{\mathrm{i}}$ \\
\hline \hline
\end{tabular}

${ }^{\mathrm{a}}$ Reference 11 .

${ }^{\mathrm{b}}$ Reference 12.

${ }^{\mathrm{c}}$ Reference 13 .

${ }^{\mathrm{d}}$ Reference 14 .

${ }^{\mathrm{e}}$ Reference 15 .

${ }^{\mathrm{f}}$ Reference 16.

${ }^{\mathrm{g}}$ Reference 17.

${ }^{\mathrm{h}}$ Reference 18 .

${ }^{\mathrm{i}}$ Reference 19.

${ }^{\mathrm{j}}$ Reference 20.

${ }^{\mathrm{k}}$ Reference 21. and $\mathrm{Al}_{2} \mathrm{O}_{3}$ are taken as a half of $D_{\text {min }}$, respectively, i.e., $0.8 \mathrm{~nm}$. Let $\kappa_{\min }=G^{\prime}\left(D_{\min } / 2\right)$ be the average thermal conductivity of $\mathrm{W}$ and $\mathrm{Al}_{2} \mathrm{O}_{3}$ with the respective intrinsic size effects at $D_{\min } / 2$, the corresponding thermal conductance $G^{\prime}=2 \kappa_{\min } / D_{\min }$, hence $\kappa_{0}=G^{\prime} D_{\min }=2 \kappa_{\min } . \kappa_{\min }$ can be calculated by $\left[\kappa_{A}(D)+\kappa_{B}(D)\right] / 2$ at $D=0.8 \mathrm{~nm}$. The intrinsically size-dependent thermal conductivity function $\kappa_{i}(D) \quad(i$ $=A, B)$ of nanoscale materials can be given as ${ }^{28}$

$$
\begin{aligned}
\kappa(D) / \kappa(\infty)= & {\left[T_{m}(D) / T_{m}(\infty)\right]^{3 / 2}=\left\{\operatorname { e x p } \left[(1-\alpha) /\left(D / D_{0}\right.\right.\right.} \\
& -1)]\}^{3 / 2},
\end{aligned}
$$

based on the kinetic formula of thermal conductivity by introducing the size effects of phonon velocity and mean free path. Because the consideration is based on the acoustic mismatch model (AMM), the interface scattering term is not included in Eq. (5).

According to Eq. (5), the calculation result shows $\kappa_{\min }$ $=1.5 \mathrm{~W} / \mathrm{m} \mathrm{K}$, and $\kappa_{0}=3 \mathrm{~W} / \mathrm{m} \mathrm{K}$, which agrees with the experimental results that the thermal conductivity of $\mathrm{Al}_{2} \mathrm{O}_{3}$ layers is $1.65 \mathrm{~W} / \mathrm{m} \mathrm{K}, 6.1 \mathrm{~W} / \mathrm{m} \mathrm{K}$ for $\mathrm{W}$ layers, ${ }^{3}$ and the average value of the both is close to $\kappa_{0}$. Applying $\kappa=\kappa_{0} t_{e}$, the thermal conductivity of $\mathrm{W} / \mathrm{Al}_{2} \mathrm{O}_{3}$ nanolaminates can be obtained as shown in Fig. 2(c). It can be seen that the theoretical prediction is in agreement with the experimental results. If the effective thermal conductance of $\mathrm{W} / \mathrm{Al}_{2} \mathrm{O}_{3}$ can be obtained by $\kappa$ divided by $D$, the calculated effective conductance is about $240 \mathrm{MW} / \mathrm{m}^{2} \mathrm{~K}$ at maximum; it keeps the 
same order at the size range of the samples. The result agrees with the experimental result of $260 \mathrm{MW} / \mathrm{m}^{2} \mathrm{~K}$ for the interface conductance of $\mathrm{W} / \mathrm{Al}_{2} \mathrm{O}_{3}^{3}$ and other measurement: ${ }^{29} 200$ and $105 \mathrm{MW} / \mathrm{m}^{2} \mathrm{~K}$ for $\mathrm{Ti} / \mathrm{Al}_{2} \mathrm{O}_{3}$ and $\mathrm{Al} / \mathrm{Al}_{2} \mathrm{O}_{3}$ interfaces, respectively, which proves that the assumption of the interface conduction dominance is reasonable.

As a comparison, the predictions based on the diffusive mismatch model $^{5}$ (DMM) are also shown in Fig. 2(c), which are larger than the experimental data. The results from the constant interface conductance show the larger error. The modification of DMM, similar to AMM, obviously improves the results. The only difference between two models is the interface phonon transmission $\left[t_{A B}=C_{B} v_{B} /\left(C_{A} v_{A}+C_{B} v_{B}\right)\right.$ based on DMM]. ${ }^{5,31}$ The better prediction based on our modified AMM may imply that the temperature effect of the thermal conductivity is weak for nanoscale structures, which is reasonable since the melting temperature decreases for nanomaterials. The temperature dependence is estimated by fitting the experimental data, ${ }^{3}$ and the result shows the smaller temperature exponent $\left[\kappa \propto f\left(T^{b}\right), b<3\right]$. Note that the interface transmission factors are both $T$ independent in two models $;^{5}$ the $T$ dependence of the interface conduction is similarly determined by the phonon distribution.

\section{CONCLUSION}

In summary, a theoretical model for size-dependent interface phonon transmission and thermal conductivity for nanolaminates is developed. The model reveals that the variety of size effect of the interface phonon transmission for different systems results from the competition of the size dependencies of phonon transport of two materials constituting the interface, interprets the variety of size dependence of thermal conductivity of different superlattices based on the interface conduction dominance proposition, and provides general guide for the application of nanoscale devices.

\section{ACKNOWLEDGMENTS}

The work is supported by grants partly from the Faculty Research Grant of NUS and DSTA Singapore under Agreement No. DSTA POD0001821 (B. Li), partly from the Chinese Academy of Sciences through Grant No. KJCX2-YWM04 and the NSFC through Grant Nos. 10721202, 10432050 .
${ }^{1}$ K. Schwab, E. A. Henriksen, J. M. Worlock, and M. L. Roukes, Nature (London) 404, 974 (2000); B. Li, L. Wang, and G. Casati, Phys. Rev. Lett. 93, 184301 (2004); T. C. Au Yeung, M. X. Gu, C. Q. Sun, G. C. K. Chen, D. W. K. Wong, and V. Nosik, Phys. Rev. B 74, 155317 (2006).

${ }^{2}$ D. G. Cahill, W. K. Ford, K. E. Goodson, G. D. Mahan, A. Majumdar, H. J. Maris, R. Merlin, and S. R. Phillpot, J. Appl. Phys. 93, 793 (2003); K. E. O'Hara, X. Y. Hu, and D. G. Cahill, ibid. 90, 4852 (2001).

${ }^{3}$ R. M. Costescu, D. G. Chill, F. H. Fabreguette, Z. A. Sechrist, and S. M. George, Science 303, 989 (2004).

${ }^{4}$ I. M. Khalatnikov, Zh. Eksp. Teor. Fiz. 22, 687 (1952).

${ }^{5}$ E. T. Swartz and R. O. Pohl, Rev. Mod. Phys. 61, 605 (1989).

${ }^{6}$ E. J. Post, Can. J. Phys. 31, 112 (1953).

${ }^{7}$ J. G. Dash, Rev. Mod. Phys. 71, 1737 (1999).

${ }^{8}$ F. G. Shi, J. Mater. Res. 5, 1307 (1994); Q. Jiang, H. X. Shi, and M. Zhao, J. Chem. Phys. 111, 2176 (1999).

${ }^{9}$ A. R. Abramson, C. L. Tien, and A. Majumdar, J. Heat Transfer 124, 963 (2002).

${ }^{10}$ Z. Zhang, M. Zhang, and Q. Jiang, Semicond. Sci. Technol. 16, L33 (2001).

${ }^{11}$ Sargent-Welch Scientific Company, Periodic Table of the Elements (Skokie, Illinois, 1980), p. 1.

${ }^{12}$ M. Winter, WebElements, the Periodic Table on the WWW, The University of Sheffield and WebElements, Ltd., UK, 1993-2006.

${ }^{13}$ D. R. Lide, Handbook of Chemistry and Physics, 85th ed. (CRC, Boca Raton, FL, 2004), pp. 12-19 and 12-21; pp. 6-125; pp. 12-98; pp. 12224.

${ }^{14}$ H. W. King, in Physical Metallurgy, 3th ed., edited by R. W. Cahn and P. Haasen (North-Holland, Amsterdam, 1983), pp. 63-65.

${ }^{15}$ http://www.cmmp.ucl.ac.uk/ ahh/research/crystal/al2o3.htm.

${ }^{16}$ ISPT Periodic Table, Qivx Inc., 2003.

${ }^{17}$ F. Szmulowicz, F. L. Madarasz, P. G. Klemens, and J. Diller, J. Appl. Phys. 66, 252 (1989).

${ }^{18}$ M. Hillert and L. I. Staffansson, Acta Chem. Scand. 24, 3618 (1970).

${ }^{19}$ S. G. Shen and X. Q. Fan, J. Phys.: Condens. Matter 9, 3151 (1997).

${ }^{20}$ H. T. Grahn, D. A. Young, H. J. Maris, J. Tauc, J. M. Hong, and T. P. Smith, Appl. Phys. Lett. 53, 2023 (1988).

${ }^{21}$ J. A. Van Vechten, Phys. Rev. B 7, 1479 (1973).

${ }^{22}$ S. M. Lee, D. G. Cahill, and R. Venkatasubramanian, Appl. Phys. Lett. 70, 2957 (1997).

${ }^{23}$ T. Borca-Tasciuc, W. Liu, J. Liu, T. Zeng, D. W. Song, C. D. Moore, G. Chen, K. L. Wang, and M. S. Goorsky, Superlattices Microstruct. 28, 199 (2000).

${ }^{24}$ S. T. Huxtable, A. R. Abramson, A. Majumdar, A. Shakouri, and E. T. Croke, Proceedings of 2002 ASME International Mechanical Engineering Congress and Exposition, New Orleans, LA, 17-22 November 2002 (unpublished), Paper No. IMECE2002-34239.

${ }^{25}$ S. Chakraborty, C. A. Kleint, A. Heinrich, C. M. Schneider, J. Schumann, M. Falke, and S. Teichert, Appl. Phys. Lett. 83, 4184 (2003).

${ }^{26}$ W. S. Capinski, H. J. Maris, T. Ruf, M. Cardona, K. Ploog, and D. S. Katzer, Phys. Rev. B 59, 8105 (1999).

${ }^{27}$ Y.-F. Chen, D.-Y. Li, J.-K. Yang, Y.-H. Wu, J. R. Lukes, and A. Majumdar, Physica B 349, 270 (2004).

${ }^{28}$ L. H. Liang and B. Li, Phys. Rev. B 73, 153303 (2006).

${ }^{29}$ R. J. Stoner and H. J. Maris, Phys. Rev. B 48, 16373 (1993).

${ }^{30}$ http://www.crystran.co.uk/products.asp?productid $=231$.

${ }^{31}$ G. Chen, Phys. Rev. B 57, 14958 (1998). 